In isolierten Chloroplasten wird die cyclische Photophosphorylierung mit Menadion durch Ascorbat stimuliert. Dies ist nicht der Fall in AnacystisPartikeln. Doch hebt Ascorbat die DCMU.Hemmung der Menadion-katalysierten Photophosphorylierung wieder auf.
Wir sind der Deutschen Forschungsge$\mathrm{me}$ in s ch a f t für ihre Unterstützung dankbar.

16 F. R. Whatley, in: Photosynthetic Mechanisms of Green Plants, National Academy of Sciences - National Research Council, Washington 1963.

\title{
Der Einfluß der Temperatur auf die Strahlenempfindlichkeit von $\mathrm{T}_{\mathbf{1}}$-Bakteriophagen
}

\author{
Klaus-Otto Hermann
}

Institut für Strahlenbiologie, Kernforschungszentrum Karlsruhe

(Z. Naturforschg. 21 b, 678-682 [1966] ; eingegangen am 8. März 1966)

\begin{abstract}
The radiosensitivity of dry bacteriophage was determined in vacuo at temperatures varying continuously between $100^{\circ}$ and $300^{\circ} \mathrm{K}$ using $2 \mathrm{MeV}$ protons. Moreover, the influence of added cystamine and of base substitution by 5 -bromouracil was investigated in the same range of temperatures. The variation of total cross section $\sigma(\mathrm{T})$ with temperature is given by the expression: $\sigma(T)=\sigma_{0} \cdot\left(1+5,3 \cdot \mathrm{e}^{-E_{\mathrm{a}} / R T} \pm 15\right.$ per cent.

The constant $\sigma_{0}$ is $3,3 \cdot 10^{-12} \mathrm{~cm}^{2}$ for normal Tl phage, $4,9 \cdot 10^{-12} \mathrm{~cm}^{2}$ for base substituted Tl phage, $1,3 \cdot 10^{-12} \mathrm{~cm}^{2}$ for $\mathrm{Tl}$ phage in presence of cystamine, and $1,6 \cdot 10^{-12} \mathrm{~cm}^{2}$ for base substituted Tl phage in presence of cystamine; $E_{\mathrm{a}}$ amounts to $1 \mathrm{kcal} / \mathrm{mole}$ in all four cases. The correlation between inactivation cross section $\sigma$ and temperature $T$ shows that two mechanisms of inactivation are present, one $\left(\sigma_{0}\right)$ being independent of temperature, the other having an activation energy of about $1 \mathrm{kcal} / \mathrm{mole}$. The first mechanism is ascribed to direct radiation action, while the temperature dependent component probably originates from small diffusible radicals.
\end{abstract}

Die Erkenntnis der zentralen Bedeutung der Nucleinsäuren für die gesamte Biologie führte zu einer in den letzten Jahren zunehmenden Zahl von Arbeiten über die Wirkung ionisierender Strahlung auf Nucleinsäuren, besonders auf DNS. Speziell bei Messungen an Bakteriophagen stellte man wiederholt fest, daß die Strahlenempfindlichkeit der DNS in starkem Maße von der während der Bestrahlung herrschenden Temperatur und der Konzentration der anwesenden Schutzstoffe abhängig ist. Durch genauere Untersuchungen des Temperatureinflusses sollte es möglich sein, die physikalischen und chemischen Mechanismen der biologischen Strahlenwirkung aufzuklären und dadurch auch neue Hinweise auf die Art der DNS-Schädigung zu gewinnen.

\section{Experimenteller Teil}

1.1. Versuchsobjekt: Die Verwendung von T1-Bakteriophagen als Untersuchungsobjekt hat folgende Gründe: T1-Phagen bestehen etwa zur Hälfte aus DNS, die Strahlenwirkung kann an ihnen ohne Störung durch physiologische Vorgänge untersucht werden, die Ergebnisse sind statistisch leicht zu sichern, die erhaltenen Dosis-Effekt-Beziehungen sind bei ionisieren- der Strahlung meist Eintrefferkurven und außerdem wird die Aktivität der Viren durch extreme Umweltsbedingungen kaum beeinträchtigt.

1.2. Probenherstellung: Es wurden jeweils $0,01 \mathrm{ml}$ Phagensuspension in Nährbouillon auf Feingoldronden $(\phi 12 \mathrm{~mm})$ aufgebracht und eingetrocknet. Durch das Trocknen verlieren zwischen $50-90 \%$ der Viren ihre Aktivität. Wird die Bouillonkonzentration von 0,8 auf $4 \%$ erhöht, so steigt der Prozentsatz der Überlebenden stark an. Bei Messungen mit Cystaminzusatz wurden deshalb die Phagen in 4\% Difco Nährbouillon suspendiert, da eine niedrige Restaktivität die Genauigkeit der Ergebnisse beeinträchtigt.

1.3. Herkunft und Herstellung der Phagen: Der untersuchte Tl-Coliphage und seine Wirtszellen rührten von Stämmen her, die uns 1958 vom Institut für Genetik der Universität Köln zur Verfügung gestellt und in unserem Institut in Passagen weitergeführt worden waren. Kulturen der Bakterienstämme wurden, ebenso wie die Lysate, nach der üblichen Methode in Difco-Nährbouillon angesetzt. Bei den Versuchen mit Schutzstoff wurde die Verdünnung der Stammsuspension in Nährbouillon durchgeführt, die außerdem $0,1 \mathrm{Mol} / \mathrm{l}$ Cystamin enthielt. Nach einer von STAHL ${ }^{1}$ angegebenen Methode wurde bei einem Teil der Phagen 5-Bromuracil anstelle von Thymin in die PhagenDNS eingebaut. Der Einbau des Bromuracils hat eine wesentliche Steigerung der Strahlenempfindlichkeit zur Folge, die vom Prozentsatz des ersetzten Thymins ab- 
hängt. UV-Bestrahlungen an den von uns verwendeten T1-BUDR-Phagen zeigten, daß etwa $65 \%$ des normalerweise vorhandenen Thymins durch Bromuracil ersetzt worden war.

1.4. Bestrahlungseinrichtung: Die Bestrahlungen wurden mit 2-MeV-Protonen eines $\mathrm{V}$ a n de G r a a f f Beschleunigers durchgeführt. Der Protonenstrahl wurde durch einen Analysiermagneten gereinigt und durch zwei Nickelfolien derartig aufgefächert, daß eine homogene Probenausleuchtung erzielt wurde. Den Aufbau der verwendeten Bestrahlungskammer zeigt Abb. 1. Der

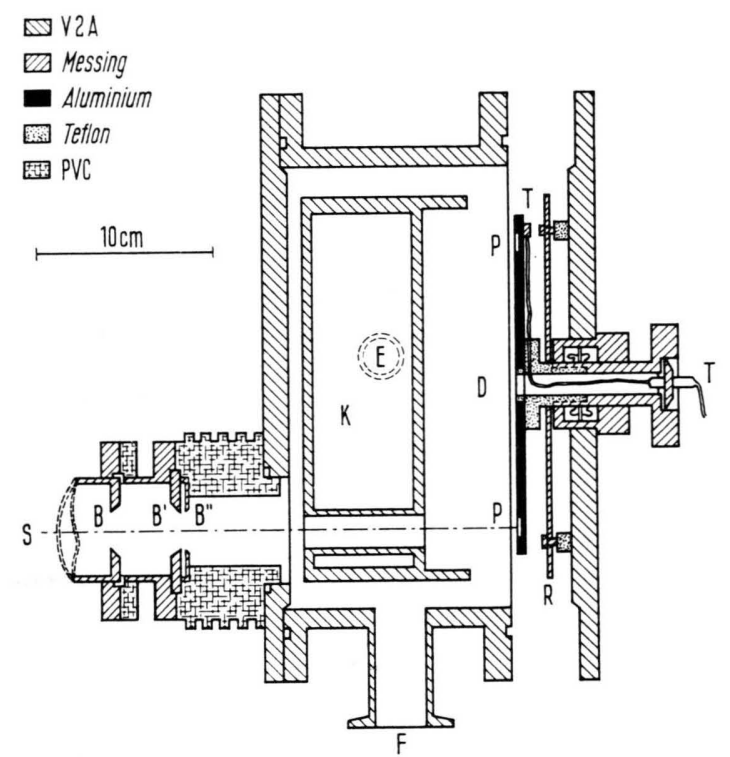

Abb. 1. Bestrahlungskammer. $\mathrm{B}=$ Eintrittsblenden, $\mathrm{D}=$ Probenhalter, $\mathrm{E}=$ Kühlmitteleinlaß, $\mathrm{F}=$ Absaugstutzen, $\mathrm{K}=$ Kühlfalle, $\mathrm{P}=$ Proben, $\mathrm{R}=$ Abdeckplatte, $\mathrm{S}=$ Strahlachse, $\mathrm{T}=$ Thermoelement.

von außen drehbare Probenhalter (D) bestand aus einer schwarzen Aluminiumscheibe mit 19 Probenpositionen. In der Kammer befand sich eine Kühlfalle $(\mathrm{K})$, die es erlaubte, Bestrahlungen bei Temperaturen bis zu $100^{\circ} \mathrm{K}$ durchzuführen. Die Kühlfalle war so konstruiert, daß der Probenhalter - bis auf die Öffnung für den Protonenstrahl - ringsum von gekühlten, schwarzen Flächen umgeben war. Bei Verwendung von flüssigem Stickstoff als Kühlmittel erreichte man innerhalb von drei Stdn. eine Abkühlung bis auf $100^{\circ} \mathrm{K}$, da sich infolge der Wärmestrahlung die Probentemperatur der Kühlmitteltemperatur anglich. Weil die Bestrahlungszeiten kurz gegen die Abkühlzeiten der Proben waren, konnte bei jeder beliebigen Zwischentemperatur bestrahlt werden.

1 F. W. Stahl, J. M. Crasemann, L. Okun, E. Fox, and C. LAIRD, Virology 13, 98 [1961].
1.5. Temperaturmessung: Da biologische Objekte sehr schlechte Wärmeleiter sind, stellt sich im Vakuum das Temperaturgleichgewicht überwiegend durch Wärmestrahlung ein. Die Temperatur des Probenhalters wurde mit einem Thermoelement gemessen, das vor jeder Bestrahlung geeicht wurde. Die Erwärmung der Proben durch die Bestrahlung berechnet sich bei einer Dosis von $1 \mathrm{Mrad}$ zu $2,4{ }^{\circ} \mathrm{K}$ (bei Zimmertemperatur) und liegt daher innerhalb der Meßfehlergrenzen. Aufheizung der Proben durch den Protonenstrahl müßte $\mathrm{zu}$ einem experimentell nicht gefundenen Steilerwerden der Dosiseffektkurven bei höheren Dosen und Dosisleistungen führen, vorausgesetzt, daß sich das Temperaturgleichgewicht nicht in so kurzer Zeit einstellt, daß diese Erwärmung noch zu den primären Energieabsorptions-Prozessen gezählt werden muß.

1.6. Bestrahlungen: Die Proben wurden zunächst einen Tag lang im Exsikkator und anschließend weitere $15 \mathrm{Stdn}$. lang in der Bestrahlungskammer bei $10^{-5}$ Torr getrocknet, ehe sie auf die gewünschte Bestrahlungstemperatur gebracht wurden. Zur Dosismetrie der 2-MeV-Protonen wurde die Kammer als $\mathrm{F}$ a r a d a y Käfig ausgebildet, und die in die Kammer gelangende Ladungsmenge mittels eines Integrators gemessen. War die vorgewählte Ladung erreicht, betätigte der Integrator einen Verschluß, der den Protonenstrahl unterbrach. Nach der Bestrahlung wurden die Proben durch Entfernung des Kühlmittels wieder auf Zimmertemperatur gebracht.

1.7. Titration der Phagen: Nach der Bestrahlung wurden die Phagen in einem Reagenzglas mit $5 \mathrm{ml}$ 1-proz. Trypton-Nährbouillon quantitativ von ihrer Unterlage abgelöst und ihre Konzentration nach konventionellen Methoden ${ }^{2}$ bestimmt.

\section{Ergebnisse}

Inaktivierungsversuche an Bakteriophagen mit ionisierender Strahlung liefern unter den meisten Versuchsbedingungen Eintrefferkurven, die sich durch die Beziehung

$$
N / N_{0}=\exp \left(-D / D_{37}\right)=\exp (-\sigma \cdot D)
$$

beschreiben lassen. Dabei bedeutet $N$ die Zahl der aktiven Phagen bei der Strahlendosis $D, N_{0}$ die Zahl der ursprünglich (bei $D=0$ ) vorhandenen Phagen, $D_{37}$ die Dosis, bei der die Zahl der aktiven Phagen auf den $e$-ten Teil der ursprünglich vorhandenen abgenommen hat, und $\sigma=1 / D_{37}$ ist der hierdurch definierte Inaktivierungs-Querschnitt. Diese Form der Dosis-Effekt-Beziehung bei Tl-Bakterio-

2 M. H. Adams, Bacteriophages, Interscience Publish Inc., New York 1959. 
phagen ist wiederholt ${ }^{3-12}$ und mit verschieden ionisierenden Strahlenarten gemessen worden. Auch unsere Messungen an normalen Tl-Phagen und TlBUDR-Phagen ergaben sowohl mit als auch ohne Cystaminzusatz gut reproduzierbare Eintrefferkurven, von denen zwei als typische Beispiele in Abb. 2 aufgetragen sind. In der Tabelle sind die unter den verschiedenen Versuchsbedingungen ermittelten Inaktivierungs-Querschnitte zusammengestellt. Um die Abhängigkeit der Inaktivierungs-Querschnitte von der Bestrahlungstemperatur genauer zu untersuchen, wurden die Proben mit jeweils der gleichen Dosis bestrahlt, wobei die Temperatur von Probe zu Probe in kontrollierter Weise verändert wurde. Aus dem Inaktivierungsgrad $N / N_{0}$ läßt sich nach Gl. (1) der Inaktivierungs-Querschnitt $\sigma$ ermitteln. Zur Beschreibung der Temperaturabhängigkeit von Inaktivierungs-Querschnitten wird in letzter Zeit öfter der Ansatz $\sigma=$ const $\cdot \exp \left(-E_{\mathrm{a}} / R T\right)$ verwandt ${ }^{13}{ }^{14}$, wobei $R$ die Gaskonstante und $T$ die absolute Temperatur bedeuten; $E_{\mathrm{a}}$ ist die Summe der Aktivierungsenergien der zur Inaktivierung der Phagen führenden chemischen Reaktionen. Der Wert von $E_{\mathrm{a}}$ läßt sich bei logarithmischer Auftragung von $\sigma$ über der reziproken Temperatur aus der Steigung auftretender Geraden unmittelbar bestimmen. Die Kurven I und II in Abb. 3 geben eine solche Darstellung der

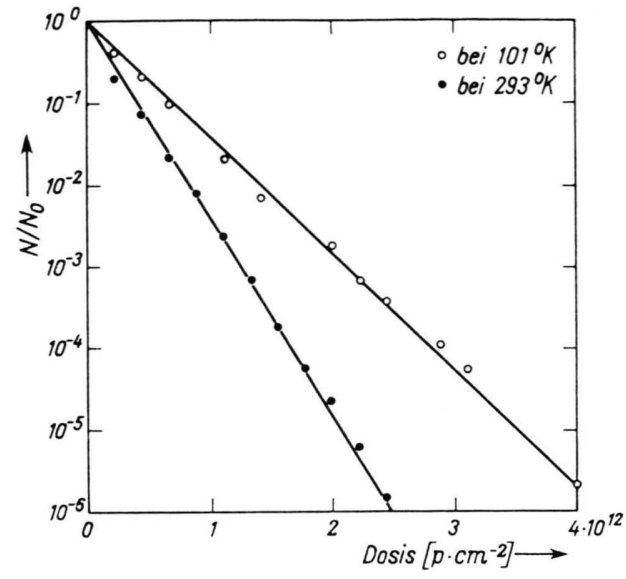

Abb. 2. Inaktivierung von Tl-Bakteriophagen durch 2-MeVProtonen bei $101^{\circ}$ und $293^{\circ} \mathrm{K}$.

an Tl-Bakteriophagen gemessenen InaktivierungsQuerschnitte wieder. Da die Kurven nicht linear sind, bedeutet dies, daß wahrscheinlich mehrere Prozesse mit unterschiedlicher Aktivierungsenergie wirksam sind. Nach Abzug eines Temperatur-unabhängigen Anteils mit einer Aktivierungsenergie $E_{\mathrm{a}}=0$ (unterbrochene Geraden in Abb. 3) ergeben sich eindeutig Arrhenius-Geraden (Kurven $\mathrm{I}_{\mathrm{a}}$ und $\mathrm{II}_{\mathrm{a}}$ in Abb. 3), deren Steigung einer Aktivierungsenergie von $1 \mathrm{kcal} / \mathrm{Mol}$ entspricht. Bei den Ver-

\begin{tabular}{|l|c|c|cc|c|}
\hline \multicolumn{1}{|c|}{ Material } & $\begin{array}{c}\text { Temperatur } \\
{\left[{ }^{\circ} \mathrm{K}\right]}\end{array}$ & $\begin{array}{c}\text { Wirkungs- } \\
\text { querschnitt } \\
{\left[10^{-12} \mathrm{~cm}^{2}\right]}\end{array}$ & \multicolumn{2}{|c|}{$\begin{array}{c}\text { Inaktivierungsdosis } D_{37} \\
{\left[\mathrm{pcm}^{-2} \cdot 10^{11}\right]}\end{array}$} & $\begin{array}{c}\text { Fehler- } \\
\text { grenzen } \\
{[\%]}\end{array}$ \\
\hline Tl & 293 & 5,41 & 1,85 & 494 & \pm 11 \\
Tl & 101 & 3,16 & 3,17 & 845 & \pm 20 \\
T1 + Cy & 293 & 2,44 & 4,09 & 1079 & \pm 14 \\
T1 + Cy & 117 & 1,76 & 5,67 & 1513 & \pm 9 \\
T1 - BUDR & 293 & 8,40 & 1,19 & 316 & \pm 12 \\
T1 - BUDR & 104 & 4,74 & 2,11 & 559 & \pm 5 \\
Tl - BUDR + Cy & 293 & 4,10 & 2,44 & 650 & \pm 12 \\
T1 - BUDR + Cy & 129 & 2,00 & 5,00 & 1335 & \pm 6 \\
\hline
\end{tabular}

Tab. 1. Wirkungsquerschnitt $\sigma$ und $D_{37}$ für die Inaktivierung von T1-Bakteriophagen mit 2-MeV-Protonen. Die angegebenen Werte wurden aus der Steigung der Ausgleichgeraden durch die Meßpunkte bestimmt. Dabei wurden die Werte der ersten Dekade $\left(N / N_{0}>0,1\right)$ wegen ihrer offensichtlichen Ungenauigkeit verworfen. Die angegebenen Fehlergrenzen sind die mit dem sog. $t$-Faktor für $5 \%$ multiplizierten Standardabweichungen dieser Regressionsanalysen.

3 W. R. Adams and E. Pollard, Arch. Biochem. Biophysics 36, $311[1952]$.

4 C. S. Bachofer, C. F. Ehret, S. Mayer, and E. L. Powers, Bacteriology 36, 744 [1953].

5 T. Brustad, Rad. Res. 15, 139 [1961].

6 M. Davis, Arch. Biochem. Biophysics 49, 417 [1954].

7 D. J. Fluke, T. Brustad, and A. Birge, Rad. Res. 13, 788 [1960].

8 D. J. Fluke and F. Forro, Rad. Res. 13, 305 [1960].
9 G. Hotz and K. G. Zimmer, Int. J. Rad. Biology 7, 75 [1963].

10 Pн. Schambra and F. Hutchinson, Rad. Res. 23, 514 [1964].

11 G. Hoтz, Habilitationsschrift, Technische Hochschule Karlsruhe (1965).

12 G. Нотz, Z. Naturforschg. 17 b, 37 [1962].

13 L. G. Augenstein, Advances in Enzymol. 24, 360 [1962].

14 L. G. Augenstein, T. Brustad, and R. Mason, Advances in Rad. Biol. 1, 228 [1964]. 


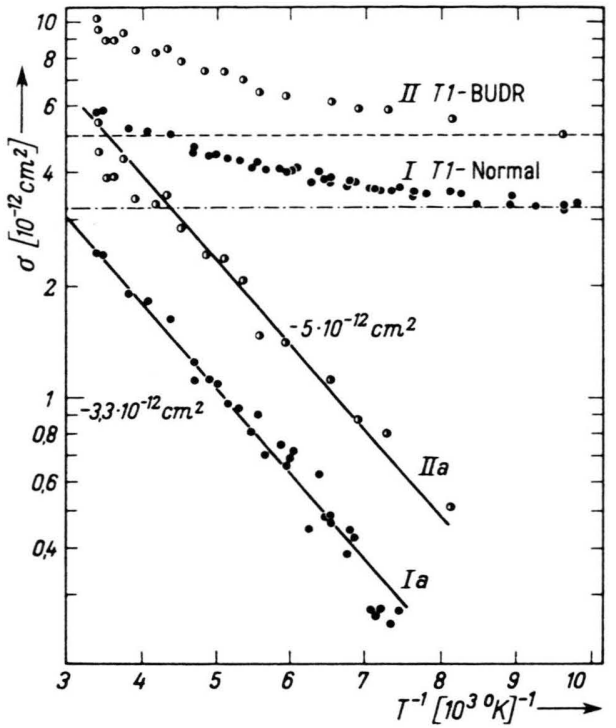

Abb. 3. Verlauf des Inaktivierungs-Querschnitts von Tl-Baktheriophagen mit der Temperatur, gemessen mit 2-MeV-Protonen. I bei normalen T1-Phagen, Ia nach Abzug eines konstanten Anteils $\sigma_{0}$ (T1), II bei schweren T1-BUDR-Phagen, IIa nach Abzug eines konstanten Anteils $\sigma_{0}$ (Tl-BUDR).

suchen mit Cystamin zeigen die gemessenen Inaktivierungs-Querschnitte das gleiche Verhalten; auch hier findet man zwei Komponenten, von denen die eine Temperatur-unabhängig ist $\left(E_{\mathrm{a}}=0\right)$, während die andere eine Aktivierungsenergie von $1 \mathrm{kcal} / \mathrm{Mol}$ besitzt. Die Abhängigkeit der unter verschiedenen Versuchsbedingungen ermittelten InaktivierungsQuerschnitte $\sigma(T)$ von der Temperatur läßt sich durch folgende Ausdrücke beschreiben:

$$
\left.\begin{array}{l}
\sigma_{1}=3,3 \cdot\left(1+4,2 \cdot \mathrm{e}^{-E_{\mathrm{a}} / R T}\right) \\
\sigma_{2}=4,9 \cdot\left(1+5,3 \cdot \mathrm{e}^{-E_{\mathrm{a}} / R T}\right) \\
\sigma_{3}=1,3 \cdot\left(1+5,8 \cdot \mathrm{e}^{-E_{\mathrm{a}} / R T}\right) \\
\sigma_{4}=1,6 \cdot\left(1+5,9 \cdot \mathrm{e}^{-E_{\mathrm{a}} / R T}\right)
\end{array}\right\} \cdot 10^{-12} \mathrm{~cm}^{2}
$$

$\operatorname{mit} E_{\mathrm{a}}=1 \mathrm{kcal} / \mathrm{Mol}$.

$\sigma_{1}$ gilt für normale T1-Phagen, $\sigma_{2}$ für T1-BUDRPhagen, $\sigma_{3}$ für normale Tl-Phagen mit Cystaminzusatz und $\sigma_{4}$ für T1-BUDR-Phagen mit Cystaminzusatz. Bemerkenswert ist, daß die Klammerausdrücke, die den Einfluß der Temperatur wiedergeben, in allen vier Gleichungen innerhalb der Fehlergrenzen etwa gleich sind. Der Schutzeffekt durch Cystamin ist bei normalen wie bei Basen-substituierten Tl-Phagen gleich; das zeigt sich darin, daß die Verhältnisse $\sigma_{1} / \sigma_{3}$ und $\sigma_{2} / \sigma_{4}$ in beiden Fäl-

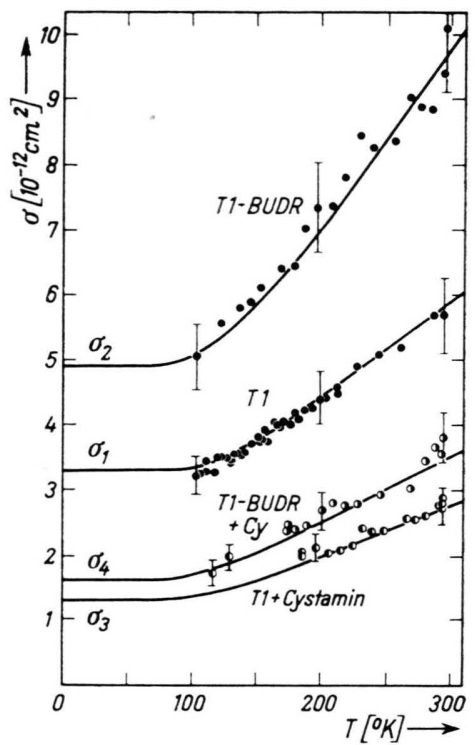

Abb. 4. Verlauf des Inaktivierungs-Querschnitts für Tl-Bakteriophagen mit der Temperatur, gemessen mit 2-MeV-Protonen. Die durchgezogenen Kurven geben den Verlauf nach Gl. (2) wieder.

len etwa 2,5 betragen. Die bei verschiedenen Temperaturen gemessenen Wirkungsquerschnitte sind in Abb. 4 über der Temperatur aufgetragen, wobei die durch die Meßpunkte gelegten Kurven Gl. (2) entsprechen. Die Abb. 4 zeigt, wie genau im Temperaturintervall zwischen 100 und $300^{\circ} \mathrm{K}$ der Verlauf des Wirkungsquerschnitts durch Gl. (2) beschrieben wird.

\section{Diskussion}

Die als Versuchsergebnis gewonnenen Gln. (2) zeigen, daß sich die beobachtete biologische Wirkung der Protonen aus zwei Komponenten zusammensetzt, von denen eine nicht von der Bestrahlungstemperatur, sondern nur von der Basenzusammensetzung und der Cystaminzugabe abhängt, während die andere durch die Temperatur zu beeinflussen ist. Bemerkenswert ist, daß die Temperaturabhängigkeit in allen vier Fällen $\left(\sigma_{1}, \sigma_{2}, \sigma_{3}, \sigma_{4}\right)$ durch Ausdrücke mit einer einheitlichen Aktivierungsenergie beschrieben wird. Auch bei Inaktivierungsversuchen an anderen biologischen Objekten, bei denen der Temperatureinfluß untersucht wurde, fand man Aktivierungsenergien dieser Größe. Aus- 
führliche Messungen an Bakteriensporen ${ }^{15-17}$ zeigten, daß der Inaktivierungs-Querschnitt zwischen 5 und $80^{\circ} \mathrm{K}$ praktisch konstant verläuft $\left(E_{\mathrm{a}}=0\right)$ und nach höheren Temperaturen hin wie bei den vorliegenden Messungen ansteigt. Unser Auswertungsverfahren ergibt auch hier ${ }^{15}$ eine Aktivierungsenergie von etwa $1 \mathrm{kcal} / \mathrm{Mol}$. An Enzymen sind Messungen des Temperaturverlaufs von Inaktivierungs-Querschnitten häufig durchgeführt worden. BRUStad ${ }^{18}$ hat die Inaktivierung von Trypsin mit schweren Ionen, F LUKE $^{19}$ die Inaktivierung von Ribonuclease und Lysozym mit 3-MeV-Elektronen gemessen. Die Inaktivierung von Ribonuclease mit 2-MeV-Protonen und Deuteronen wurde außerdem von Günther ${ }^{20}$ untersucht. Henriksen ${ }^{21}$ hat die mit der Temperatur zunehmende Radikalausbeute in Ribonuclease, Trypsin und Lysozym bei Bestrahlung mit Elektronen und schweren Ionen gemessen. In allen Fällen wurde ein konstanter $\left(E_{\mathrm{a}}=0\right)$ und ein Temperatur-abhängiger Anteil mit einer Aktivierungsenergie von etwa $1 \mathrm{kcal} / \mathrm{Mol}( \pm 40 \%)$ ermittelt.

Die Experimente werden durch die folgende Hypothese gedeutet: Wenn außer der Ionisierungsenergie genügend Energie in Form thermischer Anregung auf ein Molekül übertragen wird, kann es nach Platzman $^{22}$ zu einer Dissoziation des getroffenen Moleküls in zwei Molekülfragmente kommen. Unter Umständen führt dieser primäre Prozeß schon zu einer beobachtbaren biologischen Wirkung. Der dadurch bewirkte Anteil des Inaktivierungs-Querschnitts ist unabhängig von der Bestrahlungstemperatur, da Ionisationen und Molekülanregungen Temperatur-unabhängig sind. Viele Dissoziationen, bei denen sich kleine Radikale, zumeist wahrscheinlich Wasserstoffatome ${ }^{23}$, bilden, sind aber zunächst biologisch irrelevant. Die kurz nach ihrer Entstehung im thermischen Gleichgewicht mit ihrer Umgebung stehenden Wasserstoffradikale können verschwinden, indem sie mit anderen rekombinieren, ein neues Radikal durch Reaktion mit einem unbeschädigten Nucleotid bilden oder ohne Reaktion entweichen. Wasserstoffradikale können aber nach einer Нуро-

15 R. B. Webb, C. F. Ehret, and E. L. Powers, Experientia [Basel] 14, 324 [1958].

16 E. L. Powers, r. B. Webb, and C. F. Ehret, in: Progress in Nuclear Energy, Series VI, Vol. 2 (eds. Bugher, Coursaget, and Loutit), Pergamon Press, New York 1959, p. 189.

17 E. L. Powers, B. F. Kaleta, and R. B. WebB, Rad. Res. 11, 461 [1959].

18 T. Brustad, in: The Biological Effects of Neutron Irradiations, Intern. Atomic Energy Agency, Wien 1964.

19 D. J. Fluke, Rad. Res. 25, 18 [1965]. these von BraAms ${ }^{24}$ den Temperatur-abhängigen Anteil des Inaktivierungs-Querschnitts bewirken, da sie im Festkörper selbst bei tiefen Temperaturen diffundieren. Je nach Temperatur treten die schädigenden oder die harmlosen Reaktionen in den Vordergrund. Bei tiefen Temperaturen überwiegt wegen der geringen Energie der Wasserstoffradikale die Rekombination und das Entweichen, so daß wir nur die primären Effekte bemerken. Damit die Radikale aber eine Austausch- oder Anlagerungsreaktion durchführen können, ist ein Mindestmaß thermischer Energie, die Aktivierungsenergie, erforderlich, die für Radikalreaktionen nicht besonders groß ist. So gehen z. B. die exothermen Anlagerungsreaktionen des Wasserstoffatoms an Olefine oder andere Verbindungen mit Mehrfachbindungen (selbst bei $80^{\circ} \mathrm{K}$ ) mit Aktivierungsenergien zwischen 1 bis $4 \mathrm{kcal} / \mathrm{Mol}$ vor $\operatorname{sich}^{25}$. Mit zunehmender Temperatur wächst die Zahl der Radikale ausreichender Energie exponentiell an, weshalb wir für den InaktivierungsQuerschnitt Ausdrücke wie Gl. (2) erhalten.

Da durch Cystaminzugabe sowohl der Temperatur-abhängige als auch der Temperatur-unabhängige Anteil um den gleichen Faktor von etwa 0,4 ab. nehmen, wird offenbar durch das Cystamin die Zahl der primären Dissoziationen herabgesetzt, wodurch die Zahl der diffusiblen Radikale im gleichen Maße verringert wird.

Auf Grund unserer Experimente konnte eine Aussage über die physikalischen und chemischen Prozesse, die für die Schutzwirkung des Cystamins und den beobachteten Temperatureffekt verantwortlich sind, gemacht werden. Unsere Interpretation liefert zwanglos eine Erklärung dafür, daß der Verlauf der Inaktivierungs-Querschnitte mit der Temperatur von der Art der untersuchten biologischen Objekte weitgehend unabhängig ist.

Herrn Prof. K. G. Zimmer danke ich für die Anregung zu dieser Arbeit und sein stets förderndes Interesse, den Herren Priv. Doz. Dr. G. Hotz, Dr. H. Jung und Priv. Doz. Dr. A. Müller für Unterstützung bei der mikrobiologischen Technik und nützliche Ratschläge. Herrn P. Kindermann bin ich für seine Hilfe bei den Bestrahlungen zu großem Dank verpflichtet.

20 W. Günther, Dissertation, Heidelberg 1965.

21 T. Henriksen, University of California Radiation Laboratory Report, UCRL-16358 (1965).

22 R. L. Platzman, in: Radiation Biology and Medicine (ed. W. D. Claus), Addison-Wesley Publishing Co., Reading, Mass. 1958.

23 K. G. Zrmmer, Rad. Res. Suppl. 1, 519 [1959].

24 R. R. Braams, Nature [London] 200, 752 [1963].

25 N. N. Semuonow, Einige Probleme der chemischen Kinetik und Reaktionsfähigkeit, Akademie Verlag, Berlin 1961. 


\title{
Darstellung und Eigenschaften von Coenzym A-Thiolestern substituierter Zimtsäuren
}

\author{
G. G. Gross und M. H. ZeNK
}

Botanisches Institut der Universität München*

(Z. Naturforschg. 21 b, 683-690 [1966] ; eingegangen am 2. April 1966)

\begin{abstract}
CoA-thiolesters of substituted cinnamic acids probably occupy a central role in plant metabolism, e.g. in the biosynthesis of lignin, flavonoids, alkaloids, and many other plant products. This paper describes the preparation and some properties of a number of cinnamyol-CoA-esters. The thiolesters were prepared by the action of acyl-CoA-synthetase (E C 6.2.1.2) on the corresponding cinnamic acids, CoA, and ATP; the procedure is especially useful in preparing labelled esters. Cinnamyol-CoA, $p$-cumaroyl-CoA, and feruloyl-CoA were prepared in pure form by preparative chromatography from the incubation mixtures in 10-25\% yield. Their Rf-values, absorption spectra, difference spectra, and hydrolysis constants were determined. Furthermore, a sensitive optical test is described for following the activation of cinnamic acids; for instance, the activation of $1 \mathrm{~m} \mu \mathrm{M}$ of $p$-methoxy-cinnamic acid still can be measured with accuracy. The molar extinction coefficient, $\lambda_{\max }$ values of the difference spectra, and the rate of activation for eleven different cinnamic acids are given. The formation of conjugates between glycine and cinnamic acids proceeds via the CoA-thiolesters of these cinnamic acids.
\end{abstract}

Im Text werden folgende Abkürzungen gebraucht: Adenosintriphosphat = ATP; Coenzym A = CoA; Cytidintriphosphat = CTP; Guanosindiphosphat $=$ GDP; reduziertes Glutathion = GSH; Guanosintriphosphat = GTP; Inosintriphosphat $=$ ITP; anorganisches Phosphat $=\mathrm{P}_{\mathrm{i}}$; Tris(hydroxymethyl) -aminomethan $=$ Tris; Uridintriphosphat $=\mathrm{UTP}$.

Bei der Biosynthese einer Reihe von Pflanzeninhaltsstoffen, an denen Zimtsäuren als Vorstufen beteiligt sind, müssen intermediär „aktivierte“ Zimtsäure-Derivate gebildet werden. Auf Grund vergleichend biochemischer Überlegungen erscheint es als sehr wahrscheinlich, daß diese aktivierten Zwischenprodukte in höheren Pflanzen Coenzym A-Thiolester der Phenylacrylsäuren sind. Als wohl wichtigster Schritt, für den die Teilnahme von Zimtsäure-CoAEstern postuliert wird, dürfte die stark endergonische Reduktion der Zimtsäuren zu den Ligninvorstufen, den Zimtalkoholen, gelten ${ }^{\mathbf{1}, 2}$. Auch für die Flavonoidbiosynthese wird seit langem die Kondensation von Zimtsäure-CoA-Thiolestern mit 3 Mole-

* Adresse: 8 München 19, Menzinger Straße 67.

1 A. C. NeIsh, in: The Formation of Wood in Forest Trees (Hrsg.: M. H. Zimmermans), S. 219, Academic Press, New York-London 1964; P. Higuchi u. S. A. Brown, Can. J. Biochem. Physiol. 41, 621 [1963].

2 M. H. ZENK, 2nd Meeting of the Federation of European Biochemical Societies, im Druck [1965].

3 A. J. Bircir u. F. W. Donovan, Austral. J. Chem. 6, 360 [1953].

4 H. Grisebach, in: Chemistry and Biochemistry of Plant Pigments (Hrsg.: T. W. Goodwin), S.279, Academic Press, London-New York 1965.

5 G. Billek u. H. Kinde, Österr. Chemiker-Ztg. 63, 273 [1962]. külen Acetyl-CoA ${ }^{3}$ oder Malonyl-CoA ${ }^{4}$ gefordert. Dieser Mechanismus der Seitenketten-Verlängerung dürfte ebenso für die Biosynthese der Stilbene ${ }^{5}$ und Aurone, sowie für Piperinsäure, Cortisalin u.a. zutreffen. Die Biosynthese gewisser Amide wie Cinnamoylhistamin $^{6}, \quad p$-Cumaroylagmatin ${ }^{7}$, Feruloylputrescin ${ }^{8}$, Adenocarpin ${ }^{9}$ oder Estern wie Chlorogensäure und Sinapin (Cholinester der Sinapinsäure) muß ebenfalls über energiereiche Stufen der Zimtsäuren erfolgen.

Einen klaren Hinweis für das Auftreten von Zimtsäure-CoA-Thiolestern im pflanzlichen Stoffwechsel ergaben die Untersuchungen über den Abbau von Zimtsäuren zu Benzoesäuren ${ }^{10}$, Benzaldehyden ${ }^{11}$ und Benzalkoholen ${ }^{2}$. Dabei konnte gezeigt werden, daß die Seitenkette der Zimtsäuren um zwei Kohlenstoffatome verkürzt wird und dieses $\mathrm{C}_{2}$-Bruchstück als Acetat oder Acetyl-CoA frei wird ${ }^{12,2}$. Der Abbau der Seitenkette der Zimtsäuren folgt also formal,

6 J. S. Fitzgerald, Austral. J. Chem. 17, 375 [1964].

7 A. Stoessu, Phytochemistry 4, 973 [1965].

8 T. A. Wheaton u. J. Stewart, Nature [London] 206, 620 [1965].

9 H. R. Schütte, K. L. Kelling, D. Knöfel u. K. Mothes, Phytochemistry 3, 249 [1964].

10 H. Kindi u. G. Billex, Mh. Chem. 95, 1044 [1964]; H. GriseBACH u. K. O. Vollmer, Z. Naturforschg. 18 b, 753 [1963]; S. Z. El-Basyouni, D. Chen, R. K. Ibrahim, A. C. Neish u. G. H. N. Towers, Phytochemistry 3, 485 [1964] ; M. H. Zenk u. G. Müller, Z. Naturforschg. 19 b, 398 [1964].

11 M. H. ZENK, Z. Pflanzenphysiol. 53, 404 [1965].

12 K. O. Vollmer, H. J. Reisener u. H. Grisebach, Biochem. biophysic. Res. Commun. 21, 221 [1965]. 
soweit aus den in vivo-Versuchen geschlossen werden kann, dem klassischen Schema der $\beta$-Oxydation.

Das Auftreten von Konjugaten zwischen Glycin und verschiedenen Zimtsäuren im Urin ${ }^{13}$ läßt in Analogie zur Biosynthese der Hippur- ${ }^{14}$ und Indolacetursäure $^{15}$ auf die Existenz von Zimtsäure-CoAThiolestern auch im Tier schließen.

In dieser Arbeit sollten nun die CoA-Ester verschieden substituierter Zimtsäuren dargestellt, charakterisiert und ein optischer Test zum Nachweis ihrer Aktivierung ausgearbeitet werden. Eine chemische Synthese war dabei nicht durchführbar, da für die freien phenolischen $\mathrm{OH}-\mathrm{Gruppen}$ hydroxylierter Zimtsäuren geeignete Schutzgruppen fehlen, bei deren Abspaltung nicht auch gleichzeitig die empfindliche Thiolesterbindung zerstört wird. Die gesuchten Verbindungen sollten deshalb enzymatisch dargestellt werden, was bei der Spezifität einer derartigen Reaktion und der im Ansatz enthaltenen geringen Konzentration an Sulfhydrylverbindungen auch eine Thioladdition an die Doppelbindung der Phenylacrylsäuren vermeiden läßt. Dieses Verfahren hat zudem den Vorteil, daß die Zimtsäure-CoAEster ohne großen experimentellen Aufwand radioaktiv mit hoher spezifischer Aktivität dargestellt werden können.

Einen Hinweis auf ein die Aktivierung von Zimtsäuren bewirkendes Enzym enthielt eine Arbeit von Schachter und Taggart ${ }^{16}$. Die Autoren fanden, daß Zimtsäure durch Acyl-CoA-Synthetase (E.C. 6.2.1.2) aus Lebermitochondrien in den CoA-Thiolester überführt wird, wie die Abspaltung von Pyrophosphat aus ATP während der Reaktion erkennen ließ. Eigene Versuche zeigten nun, daß dieses Enzym alle von uns untersuchten Zimtsäuren, welche die verschiedensten Substitutionsmuster aufwiesen, in CoA-Ester überführte. Wir bedienten uns daher dieses Enzyms zur Darstellung der aktivierten Zimtsäuren. Ein entsprechendes Enzym pflanzlichen Ursprungs ist bisher noch nicht bekannt.

13 M. D. Armstrong, K. N. F. Shaw u. P. E. W Chemistry 218, 293 [1956].

14 D. Schachter u. J. V. TagGart, J. biol. Chemistry 203, 925 [1953].

15 M. H. ZENK, Z. Naturforschg. 15 b, 436 [1960].

16 D. Schachter u. J. V. Taggart, J. biol. Chemistry 208, 263 [1954].

17 H. R. MAhleR, S. J. W 204, 453 [1953].

18 C. R. Rossi u. D. M. Gibson, J. biol. Chemistry 239, 1694 [1964].

\section{Experimenteller Teil}

\section{Darstellung der Acyl-CoA-Synthetase}

Das Enzym wurde nach MAHLER et al. ${ }^{17}$ aus Rinderlebermitochondrien dargestellt. In Abänderung der Originalvorschrift refraktionierten wir jedoch in den Stufen 20-24, 24-31 und 31-35 g Ammoniumsulfat $/ 100 \mathrm{ml}$, da ein Teil des aktiven Proteins erst zwischen 31 und $35 \mathrm{~g}$ gefällt wird. Verwendet wurde die Fraktion 24-31 g Ammoniumsulfat, der noch die durch nochmaliges Refraktionieren der Seitenfraktionen gewonnene Fällung dieses Bereiches zugesetzt wurde. Da sich die anschließende Gelfällung mit $\mathrm{Al}(\mathrm{OH})_{3}$ nur schlecht reproduzieren ließ, wurde die Anreicherung nach diesen Schritten abgebrochen. Wir erhielten so ein zur Zimtsäureaktivierung genügend reines Enzympräparat, das von Acyl-CoA-Thiolase frei ist und sich - wenn nötig - nach der Methode von Rossi et al. ${ }^{18}$ weiter anreichern läßt. Als Aktivitätstest bedienten wir uns der optisch verfolgbaren Aktivierung von Sorbinsäure nach Michal und Bergmeyer ${ }^{19}$; die für die Versuche verwendete Enzymlösung besaß eine spezifische Aktivität von $17 \mathrm{mE} / \mathrm{mg}$ Protein bei $15 \mathrm{~m} \mu \mathrm{Mol}$ $\mathrm{CoA} / \mathrm{ml}$ Ansatz.

\section{Hydroxamsäuretest}

Die Aktivierung der Zimtsäuren wurde mittels des Hydroxamsäuretestes ${ }^{20}$ bestimmt. Dazu befanden sich in einem Reaktionsvolumen von $1 \mathrm{ml}$ in $\mu \mathrm{Mol}: 100$ Tris-HCl, $p_{\mathrm{H}} 7,6 ; 2,5$ Kaliumsalz der betreffenden Säure; $10 \mathrm{MgCl}_{2} ; 750$ Hydroxylamin; $20 \mathrm{GSH}$; 10 ATP; 0,25 CoA; $3 \mathrm{mg}$ Protein (spez. Aktivität abweichend von obiger Angabe $11 \mathrm{mE} / \mathrm{mg}$ Protein).

Die Reaktion wurde nach $60 \mathrm{Min}$. bei $37^{\circ}$ durch $\mathrm{Zu}$ gabe von $2,0 \mathrm{ml}$ Eisen-III-chlorid-Reagens ${ }^{21}$ abgestoppt und die Extinktion des Eisenkomplexes der betreffenden Hydroxamsäure photometrisch bei $540 \mathrm{~m} \mu$ bestimmt. Als Blindwert wurde jeweils eine Probe ohne Enzym und CoA mitgeführt. Dieses Verfahren war auf Kaffee- und Sinapinsäure nicht anwendbar, da EisenIII-chlorid bereits mit der freien Säure eine tief gefärbte Verbindung ergibt.

\section{Darstellung von Zimthydroxamsäure}

Zimthydroxamsäure wurde nach einer entsprechenden Vorschrift für Benzohydroxamsäure ${ }^{22}$ dargestellt; als Ausgangsmaterial diente Zimtsäureäthylester. Das Produkt wurde durch zweimaliges Umkristallisieren aus

19 G. Michal u. H. U. Bergmeyer, in: Methoden der enzymatischen Analyse (Hrsg.: H. U. Bergmeyer), S. 512, Verlag Chemie, Weinheim 1962.

20 F. Lipmann u. L. C. Tutrle, J. biol. Chemistry 159, 21 [1945].

21 P. Berg, in: Methods in Enzymology (Hrsg.: S. P. Colowick u. N. O. Kaplan), 5, S. 461, Academic Press, New York-London 1962.

22 C. R. Hauser u. W. B. Renfrow, in: Organic Syntheses (Hrsg.: A. H. Blatt), Coll. Vol. 2, S. 67, John Wiley and Sons, New York 1959. 
7,5-proz. Essigsäure und anschließendes Umkristallisieren aus Chloroform gereinigt. Schmp.: $118^{\circ}$.

Unter den Bedingungen des obigen Hydroxamsäuretests ermittelten wir einen Extinktionskoeffizienten $\varepsilon_{540}$ von $1,54 \cdot 10^{6}\left[\mathrm{~cm}^{2} / \mathrm{Mol}\right]$, d. h., $1 \mu \mathrm{Mol}$ Zimthydroxamsäure besitzt in einem Reaktionsvolumen von $3,0 \mathrm{ml}$ bei $540 \mathrm{~m} \mu$ eine Extinktion von 0,513; dieser Wert ist beträchtlich höher als derjenige für Acetylhydroxamsäure $(E=0,325)$. Das Maximum der Farbintensität des Eisenchloridkomplexes der Zimthydroxamsäure liegt zwischen 530 und $550 \mathrm{~m} \mu$.

Die übrigen Zimthydroxamsäuren wurden über den entsprechenden Methylester dargestellt. Dazu wurden $20 \mathrm{mg}$ der betreffenden Zimtsäure in $4 \mathrm{ml}$ Methanol + 1 Tropfen konz. Schwefelsäure 4 Stdn. am Rückflußkühler erhitzt. Das Methanol wurde abgedampft, der Rückstand in Äther aufgenommen und die nicht umgesetzte Säure mit 0,5-m. Bikarbonatlösung extrahiert. Nach Waschen mit Wasser wurde die ätherische Lösung eingeengt und der zurückbleibende Methylester mit $1 \mathrm{ml}$ salzfreier Hydroxylaminlösung ${ }^{22}$ versetzt. Nach einer Reaktionsdauer von 60 Min. wurde das Produkt angesäuert und durch präparative Chromatographie unter Verwendung der in Tab. 2 aufgeführten Lösungsmittel gereinigt. Zur quantitativen Bestimmung der substituierten Zimtsäurehydroxamate wurde der für Zimthydroxamsäure ermittelte Extinktionskoeffizient verwendet.

\section{Präparative Darstellung der Zimtsäure-CoA-Thiolester}

In einem Reaktionsansatz von $3 \mathrm{ml}$ wurden inkubiert in $\mu \mathrm{Mol}: 7,5-30$ Kaliumsalz der betreffenden Zimtsäure; 300 Tris-HCl, $p_{\mathrm{H}} 7,6 ; 40 \mathrm{MgCl}_{2} ; 60 \mathrm{ATP}$; 2,75 CoA; $20 \mathrm{mg}$ Acyl-CoA-Synthetase; Inkubationszeit : 2 Stdn. bei $30^{\circ}$.

Nach Abkühlen des Ansatzes auf $0^{\circ}$ und Abstoppen der Reaktion mit $0,3 \mathrm{ml} 2-n$. $\mathrm{HCl}$ wurde das denaturierte Protein abzentrifugiert und der Überstand zur Entfernung der nicht umgesetzten Säure mit $3 \cdot 4 \mathrm{ml}$ Äther extrahiert. Zur Isolierung der CoA-Ester wurde nun entweder die gesamte wäßrige Phase auf Whatman-3-Papier übertragen und chromatographiert oder mit $4 \cdot 3 \mathrm{ml}$ eines Phenol-Benzylalkoholgemisches $=3: 1$ ( $g: g)$ extrahiert, die phenolische Phase mit dem 4fachen Volumen Chloroform versetzt und die entstehende weiße Trübung mit $5 \cdot 0,4 \mathrm{ml}$ Wasser ausgeschüttelt. Die vereinigten wäßrigen Extrakte wurden nach $\mathrm{Ge}$ friertrocknung präparativ chromatographisch bei $2-4^{\circ}$ in folgenden Laufmitteln gereinigt (Tab. 1; Whatman3-Papier, aufsteigend) .

Gute Trennungen ergaben sich auch mit Äthanol : 1-m. Ammoniumacetat $=5: 2^{23}$ und Isopropanol : Essigsäure : $\mathrm{H}_{2} \mathrm{O}: \mathrm{NH}_{3}=50: 28: 20: 2^{25}$.

23 A. M. Micherson, Biochim. biophysica Acta [Amsterdam] 93, 71 [1964].

24 E. R. Stadtman, in: Methods in Enzymology (Hrsg.: S. P. Colowick u. N. O. Kaplan), 3, S. 931, Academic Press, New York 1957.

\begin{tabular}{|l|c|c|c|}
\hline & I & II & III \\
\hline Cinnamoyl-CoA & - & 0,55 & 0,71 \\
$p$-Cumaroyl-CoA & 0,52 & 0,50 & 0,75 \\
Feruloyl-CoA & 0,40 & 0,48 & 0,70 \\
CoA & 0,18 & 0,42 & 0,62 \\
\hline
\end{tabular}

Tab. 1. $R_{f}$-Werte von Zimtsäure-CoA-Estern. I: $n$-Butanol : Eisessig : $\mathrm{H}_{2} \mathrm{O}=5: 2: 3^{23}$, II : Isobuttersäure : $\mathrm{NH}_{3}: \mathrm{H}_{2} \mathrm{O}$ $=66: 1: 33^{24}$, III: $\mathrm{C}_{2} \mathrm{H}_{5} \mathrm{OH}: 0,1-n$. Na-Acetat, $p_{H} 4,5=$ $1: 1^{24}$.

Zur Lokalisierung und Identifizierung der Thiolester wurde das Nitroprussidreagens nach Stadtman 24 verwendet; die Esterspaltung erfolgte nach 1.c. ${ }^{26}$ in Ammoniakatmosphäre. CoA-Ester von Säuren mit freien phenolischen OH-Gruppen ergaben eine sich bei $\mathrm{Hy}$ drolyse verstärkende Gelbfärbung, während sonst die übliche „verzögerte“ Rotfärbung auftrat. Der Nachweis der phenolischen OH-Gruppen erfolgte durch Sprühen mit diazotierter Sulfanilsäure.

Die Ausbeute an reinem Thiolester betrug 10 bis 25\% des eingesetzten CoA-SH. Zur Synthese radioaktiv markierter Zimtsäure-CoA-Thiolester wird das Verhältnis von Zimtsäure-[ $\left.{ }^{14} \mathrm{C}\right] \mathrm{zu}$ CoA vorteilhaft auf $1: 1$ verringert. Auf diese experimentell einfache Weise lassen sich Thiolester aromatischer Säuren mit hoher spezifischer Aktivität gewinnen (vgl. ZENK und Gross ${ }^{27}$ ).

\section{Bestimmung des molaren Extinktionskoeffizienten der Zimtsäure-CoA-Thiolester}

Das UV-Spektrum der Zimtsäure-CoA-Ester zeigt außer dem typischen Absorptionsmaximum bei $260 \mathrm{~m} \mu$ noch einen auf die Thiolestergruppierung zurückgehenden charakteristischen Gipfel im langwelligen UV-Bereich. Die spezifische Extinktion bei dieser Wellenlänge ist wichtig für die Mengenbestimmung und wurde durch Vergleich mit dem von Michal und Bergmeyer ${ }^{19}$ angegebenen exakten Extinktionskoeffizienten von SorbylCoA $\left(\varepsilon_{300}=23,53 \cdot 10^{6} \quad\left[\mathrm{~cm}^{2} / \mathrm{Mol}\right]\right.$ ermittelt. Dazu wurde einem Versuchsansatz, der folgende Verbindungen in $\mu \mathrm{Mol}$ enthielt: $10 \mathrm{MgCl}_{2} ; 7,5-15 \mathrm{Kaliumsalz}$ der betreffenden Säuren; 15 ATP; 0,75 CoA; 1,25 mg Acyl-CoA-Synthetase; 0,1-m. Tris-HCl-Puffer, $p_{\mathrm{H}}$ 7,6 ad $3 \mathrm{ml}$ Gesamtvolumen; eine exakt mit Thiokinase und Phosphotransacetylase nach 1.c. ${ }^{19}$ bestimmte Menge an freiem CoA-SH zugesetzt. Die Extinktionszunahme im Absorptionsmaximum der Thiolesterbindung wurde bestimmt und die Reaktion bis zum Stillstand verfolgt. Aus diesen Werten wurde der molare Extinktionskoeffizient nach der Gleichung $\varepsilon=\frac{\Delta E \cdot V}{d \cdot C}(\Delta E=\mathrm{Ex}$ tinktionsdifferenz; $V=$ Testvolumen in $\mathrm{ml} ; d=$ Lichtweg in $\mathrm{cm} ; C=$ eingesetzte CoA-Menge in Mol) errechnet. Bei quantitativer Reaktion, die bei den ange-

25 R. D. Lane, K. C. rominger, D. L. Young u. F. Lynen, J. biol. Chemistry 239, 2865 [1964].

26 W. Seubert, Dissertation, München 1955.

27 M. H. Zenk u. G. G. Gross, Z. Pflanzenphysiol. 53, 356 [1965].

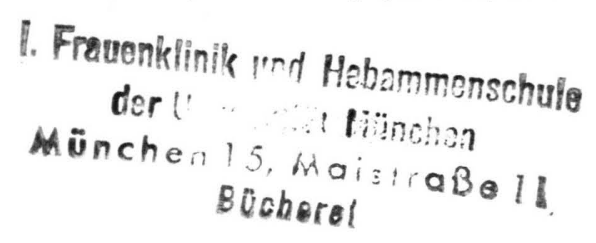


gebenen Konzentrationen der Reaktanden erfolgt ${ }^{19}$, ist die eingesetzte CoA-Menge der gebildeten Menge an Thiolester gleichzusetzen. Die Ausbildung eines Gleichgewichts zwischen den Reaktionspartnern wurde mit Sicherheit durch Zusatz einer definierten Menge an Cinnamoyl-CoA zum kompletten Ansatz ausgeschlossen; es konnte keine Verringerung der Thiolester-Konzentration festgestellt werden. Darüber hinaus wurde bei sehr langsam reagierenden Säuren eine etwa noch nicht umgesetzte Menge an CoA-SH quantitativ nach STAdTMAN ${ }^{24}$ bestimmt und dieser Wert in die Berechnung der spezifischen Extinktion mit einbezogen; der dabei auftretende Bestimmungsfehler mußte in Kauf genommen werden.

\section{Bestimmung der Hydrolysenkonstanten}

Die Hydrolysenkonstanten der gereinigten Zimtsäure-CoA-Ester wurden nach EGGERER und LyNEN ${ }^{28}$ optisch bestimmt. Dabei zeigte sich, daß besonders bei Säuren mit freien OH-Gruppen bei alkalischer $\mathrm{Hy}$ drolyse gefärbte Polymerisationsprodukte auftreten, wodurch die optische Bestimmung der Hydrolysenkonstanten und des Hydrolysenspektrums unmöglich werden. In diesen Fällen (z. B. p-Cumaroyl-CoA) wurde die Spaltung der Thiolesterbindung durch Hydroxylaminolyse bei $p_{\mathrm{H}} 7,5$ verfolgt.

\section{Konjugierung von Zimtsäuren mit Glycin}

Zum Nachweis der Konjugierung der Zimtsäuren mit Glycin im zellfreien System wurden diese mit Glycin$\left[1,2-{ }^{14} \mathrm{C}\right]$ und einer Proteinfraktion aus Rinderlebermitochondrien $(21-35 \mathrm{~g}$ Ammoniumsulfat/100 ml Rohextrakt) inkubiert, die neben Acyl-CoA-Synthetase auch Acyl-CoA: Glycin $N$-Acyl-transferase (E C 2.3.1.13) ${ }^{16}$ enthielt. Unter den in Tab. 5 erwähnten Bedingungen wurde inkubiert; nach Beendigung der Reaktion wurde das Protein mit $0,2 \mathrm{ml}$ 3-m. $\mathrm{HClO}_{4}$ ausgefällt, abzentrifugiert und der Ansatz mit $4 \cdot 1,5 \mathrm{ml}$ Äther : Äthylacetat $=6: 1$ extrahiert. Die vereinigten Ätherextrakte mit dem Zimtsäure-Glycin-Konjugat wurden mit $2 \cdot 1 \mathrm{ml}$ Wasser gewaschen, der Äther abgedampft, der Rückstand in $1 \mathrm{ml}$ abs. Methanol aufgenommen und an einem Aliquot im Methandurchflußzähler die Radioaktivität bestimmt. Durch Dünnschichtchromatographie (Kieselgel $\mathrm{GF}_{254}$ ) in verschiedenen Laufmitteln wurde nachgewiesen, daß der Extrakt frei von mitgeschlepptem Glycin war, so daß aus der spezifischen Aktivität des eingesetzten Glycins die Menge des gebildeten Acyl- $N$-Glycins berechnet werden konnte.

\section{Ergebnisse}

\section{Nachweis der Aktivierung von Zimtsäuren durch Acyl-CoA-Synthetase}

Das von uns verwendete Enzym Acyl-CoA-Synthetase besitzt einen weiten Substratbereich. So werden neben einer großen Anzahl aliphatischer

28 H. Eggerer u. F. Lynen, Biochem. Z. 335, 540 [1962].
Säuren ${ }^{17}$ auch aromatische Säuren wie Benzoe-, Phenylessig-, Nicotin-, Phenylpropion- und Zimtsäure ${ }^{16}$ sowie Indolessig-, Naphtylessig- und verschieden substituierte Phenoxyessigsäuren ${ }^{15}$ aktiviert. Es war jedoch bisher unbekannt, ob die in der Natur mit verschiedenem Substitutionsmuster auftretenden Zimtsäuren ebenfalls von diesem Enzym in die CoA-Thiolester übergeführt werden. Den Nachweis der Akitivierung konnten wir mit dem Hydroxamsäuretest führen, obwohl das zugesetzte Hydroxylamin das Enzym teilweise hemmt ${ }^{17}$. Tab. 2 zeigt die Aktivierung der verschiedenen Zimtsäuren, sowie die $R_{f}$-Werte der Zimthydroxamsäuren in

\begin{tabular}{|c|c|c|c|c|c|}
\hline \multirow{2}{*}{ Säure } & \multirow{2}{*}{$\begin{array}{c}\text { gebildete } \\
\text { Hydro- } \\
\text { xamsäure } \\
{[\mathrm{m} \mu \mathrm{Mol}]}\end{array}$} & \multirow{2}{*}[\%]{} & \multicolumn{3}{|c|}{$\begin{array}{c}R_{f} \text {-Werte der } \\
\text { Hydroxamsäuren }\end{array}$} \\
\hline & & & I & II & III \\
\hline Zimtsäure & 268 & 100 & 0,64 & 0,79 & 0,56 \\
\hline$o$-Cumarsäure & 244 & 91 & 0,50 & 0,73 & 0,29 \\
\hline$m$-Cumarsäure & 159 & 59 & - & - & - \\
\hline$p$-Cumarsäure & 120 & 45 & 0,42 & 0,66 & 0,21 \\
\hline $\begin{array}{l}p \text {-Methoxy- } \\
\text { zimtsäure }\end{array}$ & 136 & 51 & 0,47 & 0.75 & 0,46 \\
\hline Ferulasäure & 97 & 36 & 0,33 & 0,57 & 0,12 \\
\hline Isoferulasäure & 133 & 50 & 0,34 & 0,55 & 0,17 \\
\hline $\begin{array}{l}\text { 3,4-Dimethoxy- } \\
\text { zimtsäure } \\
\text { 3.4-Methylen- }\end{array}$ & 120 & 45 & 0,38 & 0,61 & 0,32 \\
\hline $\begin{array}{l}\text { dioxyzimt- } \\
\text { säure }\end{array}$ & 220 & 82 & 0,43 & 0,70 & 0,39 \\
\hline $\begin{array}{l}\text { methoxy- } \\
\text { zimtsäure }\end{array}$ & 97 & 36 & 0,53 & 0,67 & 0,43 \\
\hline
\end{tabular}

Tab. 2. Nachweis der Aktivierung verschiedener Zimtsäuren mit dem Hydroxamsäuretest. Reaktionsbedingungen wie im experimentellen Teil angegeben. Laufmittel: I 5-proz. Ameisensäure; II Butanol : Eisessig: $\mathrm{H}_{2} \mathrm{O}=20: 1: 4$; III Isopropanol : $\mathrm{NH}_{3}: \mathrm{H}_{2} \mathrm{O}=8: 1: 1$ (Schleicher \& Schüll $2043 \mathrm{~b}$, aufsteigend).

I: Butanol: Eisessig $=8: 2$; II: Benzol: Eisessig $=8: 2$; III: Toluol: Äthylformiat: Ameisensäure $=5: 4: 1$.

3 Laufmitteln. Unter den angegebenen Bedingungen ist Zimtsäure die reaktivste Säure. Es folgt $o$-Cumarsäure, während Ferula- und 3.4.5-Trimethoxyzimtsäure in relativ geringem Maße umgesetzt werden. Es steht jedoch fest, daß alle untersuchten Zimtsäuren Coenzym A-Thiolester bilden. In den Kontrollen ohne CoA oder ATP fand keine Aktivierung statt.

\section{Präparative Darstellung einiger Zimtsäure- Coenzym A-Thiolester}

Die für die Ligninbiosynthese wichtigsten Zimtsäuren sind $p$-Cumarsäure und Ferulasäure. Diese 
beiden Säuren sowie Zimtsäure sollten in ihre CoAEster überführt und präparativ gewonnen werden. Die Ansätze wurden, wie im experimentellen Teil angegeben, inkubiert und aufgearbeitet. Die Absorptionsspektren der papierchromatographisch in mindestens 2 Laufmitteln gereinigten Thiolester

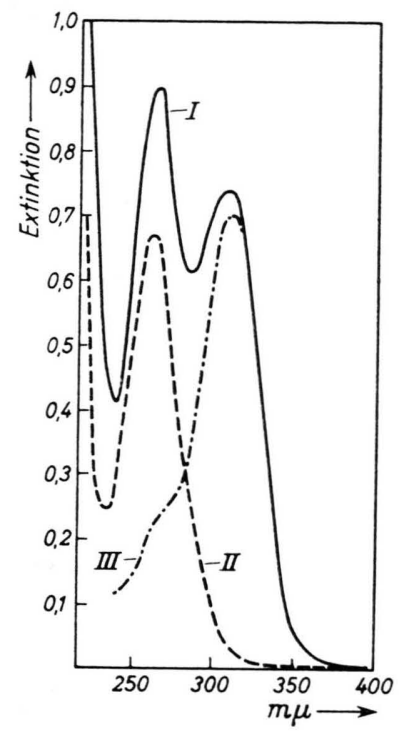

Abb. 1. UV-Spektrum von Cinnamoyl-CoA (34 m $\mu \mathrm{Mol} / \mathrm{ml})$ in $0,1-m$. Phosphatpuffer, $p_{\mathrm{H}} 7,0$ (I), Spektrum nach Hydrolyse in $0,1-m$. NaOH (II) und Differenzspektrum (III).

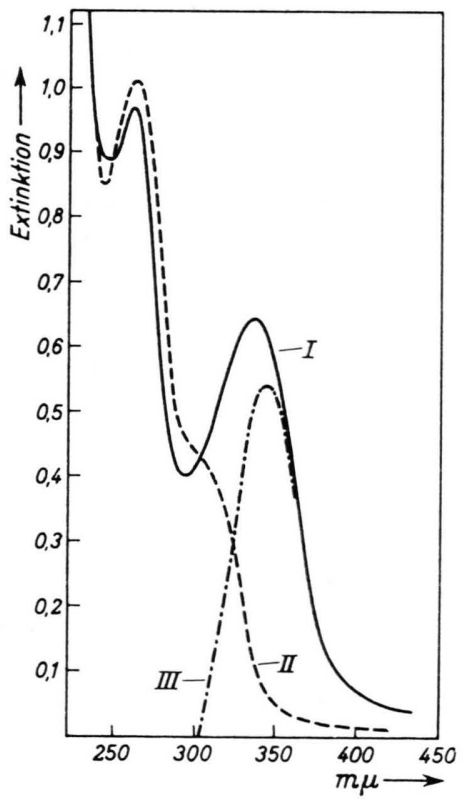

Abb. 2. UV-Spektrum von $p$-Cumaroyl-CoA $(30 \mathrm{~m} \mu \mathrm{Mol} / \mathrm{ml})$ in 0,1-m. Phosphatpuffer, $p_{H} 7,0$ (I), Spektrum nach $\mathrm{Hy}$ droxylaminolyse in $1-m$. $\mathrm{NH}_{2} \mathrm{OH}, p_{\mathrm{H}} 7,5$ (II) und Differenzspektrum (III).

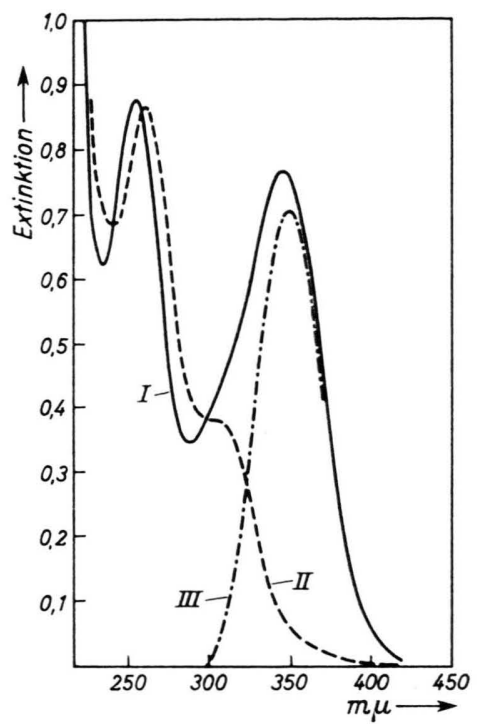

Abb. 3. UV-Spektrum von Feruloyl-CoA $(41 \mathrm{~m} \mu \mathrm{Mol} / \mathrm{ml})$ in 0,1-m. Phosphatpuffer, $p_{\mathrm{H}} 7,0$ (I), Spektrum nach Hydrolyse in $0,1-m . \mathrm{NaOH}$ (II) und Differenzspektrum (III) .

wurden in 0,1-m. K-Phosphatpuffer, $\mathrm{p}_{\mathrm{H}} 7,0$ ermittelt. In den Abbn. 1, 2, 3 sind die Absorptions-, Hydrolysen- und Differenzspektren der CoA-Verbindungen dieser drei Säuren dargestellt. Wie sich aus dem Absorptionsspektrum der gereinigten Zimtsäure-CoA-Thiolester ergab, zeigen diese Verbindungen im langwelligen UV-Bereich eine charakteristische Absorption, die bei Hydrolyse verschwindet. Im Gegensatz zu aliphatischen CoArEstern liegt das Maximum des Differenzspektrums der ZimtsäureCoA-Verbindungen nicht bei $230-240 \mathrm{~m} \mu$, sondern je nach Substitutionsmuster der Zimtsäure zwischen $310-365 \mathrm{~m} \mu$; ein ähnliches Verhalten wurde auch schon für Benzoyl-CoA ( $\Delta \mathrm{E} 272 \mathrm{~m} \mu^{14}$ ) und für Veratryl-CoA ( $\Delta \mathrm{E} 345 \mathrm{~m} \mu^{27}$ ) nachgewiesen. Zur weiteren Charakterisierung der Zimtsäure-CoA. Ester wurde die Hydrolysengeschwindigkeit von Cinnamoyl-CoA in 0.1-m. $\mathrm{NaOH}$, die Geschwindig. keit der Hydroxylaminolyse von Cinnamoyl-CoA und $p$-Cumaroyl-CoA in $1-m$. $\mathrm{NH}_{2} \mathrm{OH}, p_{\mathrm{H}} 7,5$ sowie die zugehörigen Halbwertszeiten der Esterspaltung bestimmt. Im einzelnen ergaben sich folgende Werte:

Cinnamoyl-CoA: $K_{\mathrm{NaOH}}=0,44$ [Min. $\left.^{-1}\right] ; \quad t \frac{1}{2}=$ 75 Sek., Cinnamoyl-CoA: $K_{\mathrm{NH} 2 \mathrm{OH}}=0,38$ [Min. $^{-1}$ ] ; $t \frac{1}{2}=120$ Sek., $\quad p$-Cumaroyl-CoA: $\quad K_{\mathrm{NH} 2 \mathrm{OH}}=0,18$ $\left[\right.$ Min. $\left.^{-1}\right] ; t \frac{1}{2}=240$ Sekunden.

Die graphische Darstellung der nach l.c. ${ }^{28}$ errechneten Meßwerte zeigt Abb. 4. 


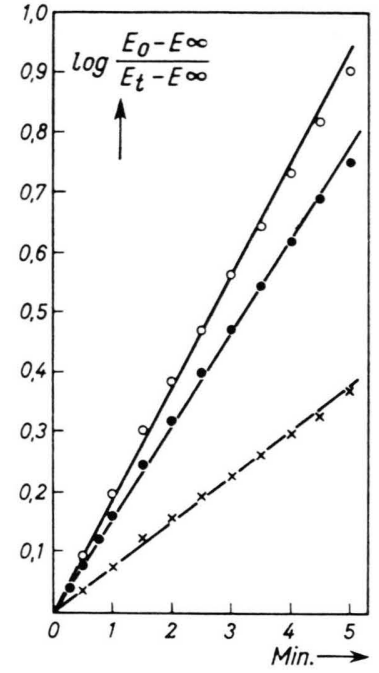

Abb. 4. Graphische Darstellung der Thiolesterspaltung. $(\mathrm{O}-\mathrm{O})$ Hydrolyse von Cinnamoyl-CoA in $0,1-m . \mathrm{NaOH}$; (-) Hydroxylaminolyse von Cinnamoyl-CoA; $(+-+)$ Hydroxylaminolyse von $p$-Cumaroyl-CoA, beide in $1-m$. $\mathrm{NH}_{2} \mathrm{OH}, p_{\mathrm{H}} 7,5 ; E_{0}=$ Anfangsextinktion, $E_{\mathrm{t}}=$ Extinktion nach $t$ Min. Hydrolyse; $E_{\infty}=$ Extinktion nach vollständiger Hydrolyse.

\section{Optischer Test zum Nachweis der Aktivierung der Zimtsäuren}

Die Absorption der Thiolesterbande der verschieden substituierten Zimtsäuren zwischen 310 und $365 \mathrm{~m} \mu$ ermöglicht einen empfindlichen optischen Test zum Nachweis ihrer Aktivierung. Dazu wird in Quarzküvetten mit den im experimentellen Teil erwähnten Reagenzien die Reaktion durch Zugabe von 1,25 mg Acyl-CoA-Synthetase gestartet. Die Extinktionszunahme bei der Wellenlänge des Absorptionsmaximums des Differenzspektrums wird bis zum Stillstand verfolgt, was je nach Säure zwischen 15 Min. (Zimtsäure) und 80 Min. (Ferulasäure) dauern kann. Als Vergleichsküvette dient ein Ansatz, der lediglich die Säure enthält, da keine andere Komponente des Inkubationsgemischs im fraglichen UV-Bereich absorbiert. Die Auswertung kann nun entweder nach der Endwertmethode oder auf kinetischer Basis erfolgen. Die Reaktion ist, nach beiden Methoden ausgewertet und mit 3.4-Methylendioxyzimtsäure als Substrat, für CoA-Mengen in einem von uns untersuchten Bereich zwischen 8 und $150 \mathrm{~m} \mu \mathrm{Mol} / \mathrm{ml}$ und für Proteinmengen zwischen 9 und $500 \mu \mathrm{g} / \mathrm{ml}$ linear. Die Thiolesterbildung steigt linear mit dem $p_{\mathrm{H}}$-Wert an (untersuchter Bereich $p_{\mathrm{H}}$ 7,4-9,0; Tris-HCl-Puffer); jedoch ist bei $p_{\mathrm{H}^{-}}$
Werten über 8 mit der Oxydation der freien -SHGruppe des CoA zu rechnen.

Von starkem Einfluß auf die Reaktionsgeschwindigkeit ist die gewählte Säurekonzentration. $10 \mu \mathrm{Mol}$ pro ml Testansatz ergeben - mit Ausnahme von $m$-Cumarsäure und 3.4-Methylendioxyzimtsäure deutliche Substrathemmung, die im Falle der Ferulasäure bis zu $80 \%$ beträgt. Abb. 5 zeigt die Konzentrations-Abhängigkeit der Umsatzgeschwindigkeit für 3.4-Methylendioxyzimtsäure und $p$-Cumarsäure. Die Optima liegen bei allen untersuchten Säuren bei ca. $2,5 \mu \mathrm{Mol} / \mathrm{ml}$.

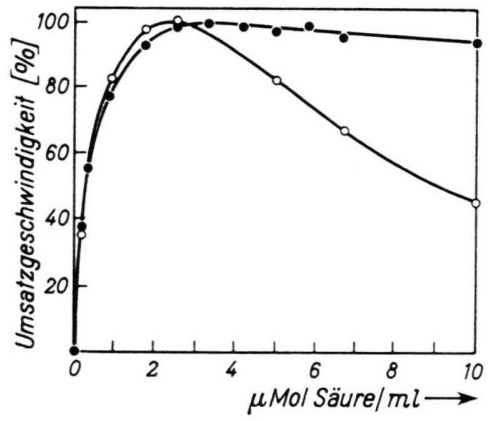

Abb. 5. Abhängigkeit der Bildungsgeschwindigkeit der Cinnamoyl-CoA-Ester von der Substratkonzentration. $(\bullet-\bullet)$ 3.4Methylendioxyzimtsäure; $(0-0) p$-Cumarsäure.

Wie Rossi et al. ${ }^{18}$ gezeigt haben, findet in Lebermitochondrien-Extrakten auch eine GTP-abhängige Aktivierung von Fettsäuren statt, wobei als Endprodukt die Acyl-CoA-Thiolester sowie GDP und $\mathrm{P}_{\mathrm{i}}$ auftreten. Daher wurde die Aktivierung von Zimtsäure und 3.4-Methylendioxyzimtsäure in Abhängigkeit von verschiedenen Nucleotidtriphosphaten untersucht. ATP erwies sich als das bei weitem wirksamste Coenzym (Tab. 3).

Der optische Test mit ATP ist so empfindlich, daß z. B. noch die Bildung von $0,96 \mathrm{~m} \mu \mathrm{Mol} p$-Methoxy-

\begin{tabular}{|c|c|r|r|r|}
\hline $\begin{array}{c}\text { Nucleotid- } \\
\text { triphosphat }\end{array}$ & $\begin{array}{c}\text { Cinnamoyl- } \\
\text { CoA } \\
{[\mathrm{m} \mu \text { Mol }} \\
\left.\cdot \text { Min. }^{-1}\right]\end{array}$ & {$[\%]$} & $\begin{array}{c}\text { 3.4-Methylen- } \\
\text { dioxycinna- } \\
\text { moyl-CoA } \\
\text { [m } \mu \text { Mol } \\
\left.\text { Min. }^{-1}\right]\end{array}$ & {$[\%]$} \\
\hline ATP & 13,5 & 100 & 9,2 & 100 \\
CTP & 0,2 & 1 & 0 & 0 \\
GTP & 1,6 & 12 & 0 & 0 \\
ITP & 2,7 & 20 & 0,7 & 8 \\
UTP & 1,0 & 7 & 0,6 & 7 \\
\hline
\end{tabular}

Tab. 3. Nucleotid-Abhängigkeit der Thiolesterbildung im optischen Test. Ansatz in $\mu \mathrm{Mol}: 10 \mathrm{MgCl}_{2} ; 30$ Kaliumsalz der betr. Säure; 15 Nucleotid; 0,25 CoA; 1,25 mg Acyl-CoA-Synthetase; 0,1-m. Tris-HCl, $p_{\mathrm{H}} 7,6$ ad $3 \mathrm{ml}$ Gesamtvolumen. 
cinnamoyl-CoA, entsprechend einer Extinktionszunahme von $E=0,025$ verläßlich gemessen werden kann. In Tab. 4 sind die von uns nach der im experimentellen Teil beschriebenen Methode gewonnenen molaren Extinktionskoeffizienten und die $\lambda_{\max }$ Werte der Thiolesterbande der Zimtsäuren zusammengestellt. Es fällt auf, daß die Absorption der Thiolesterbande bei zunehmender Kernsubstitution weiter in den langwelligen UV-Bereich verschoben

\begin{tabular}{|c|c|c|c|c|}
\hline CoA-Ester der & $\begin{array}{l}\text { Kern- } \\
\text { substitu- } \\
\text { tion }\end{array}$ & $\begin{array}{l}i \max \\
{[\mathrm{m} \mu]}\end{array}$ & $\begin{array}{c}\varepsilon \lambda_{\max } \\
{\left[\frac{\mathrm{cm}^{2}}{\mathrm{Mol}}\right] \cdot 10^{6}}\end{array}$ & $\begin{array}{c}\text { Umsatz- } \\
\text { geschwin- } \\
\text { digkeit } \\
{[\mathrm{m} \mu \mathrm{Mol}} \\
\left.\cdot \text { Min. }^{-1}\right]\end{array}$ \\
\hline $\begin{array}{l}\text { Zimtsäure } \\
o \text {-Cumarsäure } \\
m \text {-Cumarsäure } \\
p \text {-Cumarsäure } \\
p \text {-Methoxy- } \\
\text { zimtsäure } \\
\text { Kaffeesäure } \\
\text { Ferulasäure } \\
\text { Isoferulasäure } \\
\text { 3,4-Dimethoxy- } \\
\text { zimtsäure } \\
\text { 3,4-Methylen- } \\
\text { dioxyzimtsäure } \\
\text { 3,4,5-Trime- } \\
\text { thoxyzimtsäure }\end{array}$ & $\begin{array}{c}2-\overline{\mathrm{OH}} \\
3-\mathrm{OH} \\
4-\mathrm{OH} \\
\\
4-\mathrm{OCH}_{3} \\
3.4-\mathrm{OH} \\
4-\mathrm{OH} \\
3-\mathrm{OCH}_{3} \\
3-\mathrm{OH}^{2} \\
4-\mathrm{OCH}_{3} \\
3.4-\mathrm{OCH}_{3} \\
\\
3.4-\mathrm{O}_{2} \mathrm{CH}_{2} \\
\\
3.4 .5-\mathrm{OCH}_{3}\end{array}$ & $\begin{array}{l}346 \\
355 \\
342\end{array}$ & $\begin{array}{l}22 \\
\overline{11} \\
23 \\
\\
27 \\
13 \\
19 \\
18\end{array}$ & $\begin{array}{r}13,5 \\
- \\
5,5 \\
1,0 \\
11,2 \\
1,9 \\
0,1 \\
3,7\end{array}$ \\
\hline
\end{tabular}

Tab. 4. Absorptionsmaxima, molare Extinktionskoeffizienten und Bildungsgeschwindigkeiten von Zimtsäure-CoA-Thiolestern.

wird (Zimtsäure $\rightarrow p$-Cumarsäure $\rightarrow$ Kaffeesäure) . Durch $p$-Hydroxylierung wird die Umsatzgeschwindigkeit der Substrate gegenüber dem unsubstituierten Ring erheblich herabgesetzt. Ist die $p$-ständige phenolische Gruppe jedoch methyliert, steigt die Umsatzgeschwindigkeit wieder stark an. Ein ähnliches Verhalten zeigen die Isomeren Ferula- und Isoferulasäure. Hier wird die Verbindung mit methoxylierter $p$-Stellung mindestens $35 \mathrm{mal}$ schneller umgesetzt.

Noch stärkere Hemmwirkung zeigen trisubstituierte Zimtsäuren. Sinapinsäure wird z. B. so langsam umgesetzt, daß eine Bestimmung des molaren Extinktionskoeffizienten nicht möglich war. Abweichend verhält sich $o$-Cumarsäure, die zwar, wie auch schon aus dem Hydroxamsäuretest hervorgeht, sehr gut umgesetzt wird, deren CoA-Thiolester jedoch enzymatisch zu einer Verbindung weiterreagiert, die wir wohl isolieren, aber noch nicht identifizieren konnten; es handelt sich bei dieser Substanz nicht um Cumarin.

\section{Konjugierung von Zimtsäuren mit Glycin}

Das Auftreten von Glycin-Derivaten der Zimtsäuren im Urin läßt, wie bereits in der Einleitung erwähnt, auf das intermediäre Auftreten von Zimtsäure-CoA-Estern schließen. Dabei dürften die so aktivierten Zimtsäuren enzymatisch mittels AcylCoA : Glycin $N$-Acyltransferase auf Glycin übertragen werden.

Eine Inkubation von Zimtsäuren unter den im experimentellen Teil dargestellten Bedingungen bewies nun, daß in vitro die aktivierten Zimtsäuren mit Glycin konjugiert werden können. In Tab. 5 sind die Umsatzraten der einzelnen Zimtsäuren mit Glycin, wie auch die $R_{f}$-Werte der so markiert gewonnenen N-Glycin-Derivate dargestellt.

\begin{tabular}{|c|c|c|c|c|c|}
\hline \multirow[t]{2}{*}{ Säure } & \multirow{2}{*}{$\begin{array}{c}\text { gebildetes } \\
\text { Acyl- } N \text { - } \\
\text { Glycin } \\
{[\mathrm{m} \mu \mathrm{Mol}]}\end{array}$} & \multirow{2}{*}[\%]{} & \multicolumn{3}{|c|}{$\begin{array}{l}R_{f} \text {-Werte der } \\
\text { Glycin-Derivate }\end{array}$} \\
\hline & & & I & II & III \\
\hline Zimtsäure & 570 & 100 & 0,78 & 0,25 & 0,48 \\
\hline$o$-Cumarsäure & 10 & 2 & 0,72 & 0,63 & 0,67 \\
\hline$p$-Cumarsäure & 120 & 21 & 0,86 & 0,17 & 0,27 \\
\hline zimtsäure & 124 & 22 & 0,83 & 0,65 & 0,49 \\
\hline Kaffeesäure & 170 & 30 & 0,17 & 0,26 & 0,31 \\
\hline Ferulasäure & 20 & 4 & 0,79 & 0,11 & 0,29 \\
\hline Isoferulasäure & 10 & 2 & - & 0,43 & - \\
\hline $\begin{array}{l}\text { 3.4-Dimethoxy- } \\
\text { zimtsäure }\end{array}$ & 12 & 2 & 0,09 & 0,57 & 0,98 \\
\hline $\begin{array}{l}\text { 3.4-Metnylen- } \\
\text { dioxyzimtsäure }\end{array}$ & 216 & 40 & 0,80 & 0,70 & 0,50 \\
\hline Sinapinsäure & 6 & 1 & - & - & - \\
\hline $\begin{array}{l}3.4 .5 \text {-Trime- } \\
\text { thoxyzimtsäure }\end{array}$ & 18 & 3 & 0,80 & 0,27 & 0,41 \\
\hline
\end{tabular}

Tab. 5. Bildung von Zimtsäure- $N$-Glycin-Konjugaten. Ansatz in $\mu \mathrm{Mol}: 100$ Tris- $\mathrm{HCl}, p_{\mathrm{H}} 8,0 ; 10$ Kaliumsalz der betr. Säure; 50 Glycin- $\left[1.2-{ }^{14} \mathrm{C}\right]=2 \mu \mathrm{C} ; 3 \mathrm{MgCl}_{2} ; 10 \mathrm{GSH} ; 10$ ATP; $0,33 \mathrm{CoA} ; 5 \mathrm{mg}$ Protein; Vol. $1 \mathrm{ml}$; Inkubation $2 \mathrm{Stdn}$. bei $38{ }^{\circ} \mathrm{C}$.

Kontrollen ohne CoA oder ATP ergeben im Ätherextrakt des Inkubationsansatzes keine Radioaktivität. Es wird auch hier wieder die Zimtsäure ohne Kernsubstitution am besten umgesetzt. Erstaunlich ist die hohe Umsatzrate der Kaffeesäure in diesem System. Auch Sinapinsäure wird in ein Glycin-Derivat überführt, allerdings etwa 100-mal geringer als Zimtsäure; die $R_{f}$-Werte dieses Sinapinoylglycins konnten wegen der geringen Radioaktivität nicht ermittelt werden. Aus diesem Ver- 
such kann geschlossen werden, daß sich auch die Konjugierung der Zimtsäuren nach dem Schema der Hippursäure-Biosynthese vollzieht. Es ist somit gesichert, $\mathrm{da} \beta$ in vivo - zumindest im tierischen Organismus - Zimtsäure-CoA-Thiolester als energiereiche $\mathrm{Zwischenprodukte} \mathrm{auftreten.}$

Durch die Möglichkeit, die CoA-Thiolester substituierter Zimtsäuren darzustellen oder sie mit einem enzymatischen Hilfssystem zu erzeugen, kann nun geprüft werden, ob durch Pflanzenhomogenate die in der Einleitung erwähnten Biosyntheseschritte katalysiert werden können. Insbesondere ist dies von Interesse bei Untersuchungen zur Frage der mög- lichen Reduktion dieser energiereichen Verbindungen zu den Ligninvorstufen, den Zimtaldehyden und -alkoholen. Weiterhin kann mit Hilfe des beschriebenen optischen Tests entschieden werden, ob Zimtsäuren auch in höheren Pflanzen nach dem beschriebenen Reaktionsmechanismus aktiviert werden, oder ob dies möglicherweise auf dem von uns bei Basidiomyceten gefundenen Wege ${ }^{27}$ geschieht.

Fräulein C. Kalteis danken wir sehr herzlich für ihre sorgfältige Mitarbeit und der Deutschen Forschungsgemeins ch af t für die großzügige Unterstützung dieser Arbeit.

\title{
Über die osmiophilen globulären Lipideinschlüsse der Chloroplasten
}

\author{
Hartmut K. Lichtenthaler und Benno Sprey \\ Botanisches Institut der Westfälischen Wilhelms-Universität Münster
}

(Z. Naturforschg. 21 b, 690-697 [1966] ; eingegangen am 6. April 1966)

\begin{abstract}
The electromicroscopical structure and quinone composition of the osmiophilic, globular, lipiderich plastid inclusions were investigated in several plants. The localisation of the osmiophilic globuli in the plastid stroma and their behaviour toward fixation agents are described. The isolated globuli - here termed plastoglobuli - contain high amounts of lipophilic chloroplasts quinones and apparently no chlorophylls or carotenoids. There exist considerable differences in the relative quinone concentrations of chloroplasts and isolated plastoglobuli. The possible function of plastoglobuli is discussed.
\end{abstract}

Funktionstüchtige Chloroplasten normalgrüner Blätter enthalten eine lamellare Phase, bestehend aus Thylakoiden ${ }^{1}$, die in eine chlorophyllfreie Phase, dem proteinhaltigen Stromaplasma, eingebettet ist und gegen das umgebende Cytoplasma durch die doppelte Chloroplastenmembran (aus zwei „unit membranes“ im Sinne Robertsons ${ }^{2}$ bestehend) begrenzt wird. Das Thylakoid-freie Stromaplasma gibt nach $\mathrm{OsO}_{4}$-Fixation globuläre, stark Elektronenstreuende Einschlüsse - allgemein als osmiophile Globuli bezeichnet - zu erkennen. Diese treten solitär im Stroma auf, sind aber häufig auch in Gruppen mit hexagonaler Anordnung ${ }^{3}$ anzutreffen. Die globulären osmiophilen Einschlüsse stellen eine natürliche Chloroplastenkomponente dar. Sie kom-

1 W. MENKE, Z. Naturforschg. 16 b, 334 [1961].

2 J. D. Robertson, in: Cellular Membranes in Development, M. Locke ed., Academic Press, New York and London 1964, p. 1.

3 P. Sitte, Protoplasma 56, 197 [1963].

4 S. Murakami and A. Takamiya, in: Electron Microscopy, 5th International Congress for Electron Microscopy, Philadelphia, S. Breese ed., Academic Press, New York and London 1962, Vol. 2, XX 12. men in den Chromatophoren der Eukaryonten verschiedener taxonomischer Stellung sowie in den unterschiedlichen Differenzierungsstadien der Chloro-, Chromo- und Leukoplasten vor. Bisher wurden sie aus den Chloroplasten nur weniger Pflanzen isoliert ${ }^{4-7}$. Sie besitzen einen hohen Lipidgehalt und enthalten u. a. die lipophilen Plastidenchinone und sehr wahrscheinlich auch Carotinoide ${ }^{4,7}$. Ihre genaue chemische Zusammensetzung, Genese und mögliche Funktion im Chloroplasten ist noch nicht bekannt. Hier berichten wir über Größe, Struktur und Chinonzusammensetzung der osmiophilen Globuli aus Chloroplasten normalgrüner Blätter verschiedener Pflanzen. Ihre mögliche Funktion im Zuge der Thylakoidsynthese wird diskutiert.

5 A. D. Greenwood, R. M. Leech, and J. P. Williams, Biochim. biophysica Acta [Amsterdam] 78, 148 [1963].

${ }^{6}$ J. L. B BILey and A. G. Whyвorn, Biochim. biophysica Acta [Amsterdam] 78, 163 [1963].

7 H. K. Lichtenthaler, Ber. dtsch. bot. Ges. 77, 398 [1964]. 